\title{
NO PLIF Imaging in the CUBRC 48” Shock Tunnel
}

\author{
N. Jiang ${ }^{1}$, J. Bruzzese ${ }^{2}$, R. Patton ${ }^{2}$, J. Sutton ${ }^{3}$, and W. Lempert ${ }^{3}$ \\ Departments of Mechanical and Aerospace Engineering \\ The Ohio State University, Columbus, $\mathrm{OH} 43201$ \\ J. D. Miller ${ }^{2}$ and T. R. Meyer ${ }^{4}$ \\ Department of Mechanical Engineering, Iowa State University, Ames, IA 50011 \\ R. Parker ${ }^{5}$, T. Wadham ${ }^{6}$ and M. Holden ${ }^{7}$ \\ 4455 Genesee Street, CUBRC, Buffalo, NY 14225 \\ and \\ P. M. Danehy ${ }^{8}$ \\ NASA Langley Research Center, Hampton, VA 23681
}

Nitric Oxide Planar Laser-Induced Fluorescence (NO PLIF) imaging is demonstrated at a $10 \mathrm{kHz}$ repetition rate in the Calspan-University at Buffalo Research Center's (CUBRC) 48-inch Mach 9 hypervelocity shock tunnel using a pulse burst laser-based high frame rate imaging system. Sequences of up to ten images are obtained internal to a supersonic combustor model, located within the shock tunnel, during a single $\sim 10$ millisecond duration run of the ground test facility. This represents over an order of magnitude improvement in data rate from previous PLIF-based diagnostic approaches. Comparison with a preliminary CFD simulation shows good overall qualitative agreement between the prediction of the mean NO density field and the observed PLIF image intensity, averaged over forty individual images obtained during several facility runs.

${ }^{1}$ Research Associate, Senior Member AIAA

${ }^{2}$ Graduate Research Assistant, Student Member AIAA

${ }^{3}$ Professor, Associate Fellow AIAA

${ }^{4}$ Assistant Professor, Associate Fellow AIAA

${ }^{5}$ Senior Research Scientist, Member AIAA

${ }^{6}$ Senior Research Scientist, Senior Member AIAA

${ }^{7}$ Program Manager, Fellow AIAA

${ }^{8}$ Research Scientist, Associate Fellow AIAA 


\section{INTRODUCTION}

The need for optical diagnostic methods suitable for application in high-enthalpy hypervelocity short-duration facilities is especially acute due to the unique capabilities of these facilities for large-scale testing of hypersonic propulsion technologies at duplicated flight conditions as well as the fundamental physics of hypervelocity flows. However, while the development of non-intrusive diagnostic techniques has experienced much recent progress, several challenges remain due to the inherent constraint of limited optical access and typically short (order 100s of microseconds to tens of milliseconds) steady-state run times. Limitations on the number of facility "shots" per day (typically three in the current facility) is another consideration. Despite these challenges, several studies incorporating advanced optical diagnostic methods in such facilities have been recently reported. For example, nitric oxide Molecular Tagging Velocimetry (NO MTV) [1], Doppler-shift based velocimetry [2,3], and NO Planar Laser-Induced Fluorescence (PLIF) measurements of number density and thermometry [4] have been performed in the Australia National University's T2 free-piston shock tunnel. More recently, two-component Doppler-shift based velocimetry has been demonstrated in the University of New South Wales' T-ADFA free-piston shock tunnel [5]. As another example, a novel fuel plume imaging diagnostic, utilizing Mie scattering from in-situ generated silica particles in combination with a long (100 microsecond) pulsed Alexanderite laser, has been developed and applied to injector studies in the NASA-HYPULSE facility at General Applied Sciences Laboratory (GASL) [6]. NO number density measurements by UV absorption and NO PLIF have also been reported in the HYPULSE facility [7]. Several diagnostic advances have also been reported in studies at the Calspan University at Buffalo Research Center (CUBRC) LENS I and LENS II Hypersonic Shock Tunnels, including temperature and velocity by water vapor-based diode laser absorption [8], NO number density, temperature and velocity by NObased diode laser absorption [9,10], and aero-optic distortion by Phase Shift Interferometry, infrared scene generation and recording techniques [11,12]. Finally, Barker et al. have reported a novel flow tagging velocimetry diagnostic for use in high enthalpy short-duration facilities based on laser enhanced ionization of atomic sodium [13], Allen, et al. have reported single laser pulse NO PLIF based thermometry in a high enthalpy shock tunnel facility located at PSI corporation [14], and McMillan et al have reported two-line NO PLIF temperature imaging of in a transverse jet in a supersonic cross flow [15].

More generally, since its inception more than twenty-five years ago [16, 17] PLIF imaging has been developed into a powerful technique for qualitative and quantitative visualization in a wide variety of flow and combustion environments. However the ability to capture unsteady flow dynamics at high speed is severely constrained by the relatively low repetition rates (order $10 \mathrm{~Hz}$ ) of typical PLIF systems. Also such instrumentation could only acquire a single image in one shock tunnel run. We have been developing, over the last several years, an ultra-high frame rate PLIF imaging system (up to $1 \mathrm{MHz}$ frame rate) which combines a "burst mode" Nd:YAGbased tunable OPO system $[18,19]$, with a variety of high frame rate cameras, including CCD, ICCD, and intensified CMOS. In addition to enabling capture of the temporal dynamics of hypervelocity flows, by operation at frame rates of the order of $10-20 \mathrm{kHz}$, the burst mode imaging systems provides the ability to obtain sets of $\sim 10$ full planar images (albeit temporally uncorrelated) in a single short-duration facility test. The work reported here was motivated by such a desire. Specifically, we demonstrate the ability to obtain ten high resolution NO PLIF images during a single run of the CUBRC 48" shock tunnel, operating at nominal Mach 9 free 
stream conditions. Images of injector plume mixing are obtained in a large-scale Multidisciplinary University Research Initiative (MURI) high-speed combustion duct in which $\mathrm{NO}$ seeded helium is injected into a pure $\mathrm{N}_{2}$ free stream. Comparison to a somewhat preliminary CFD simulation shows good overall agreement in fuel penetration and downstream spread.

\section{EXPERIMENTAL}

\subsection{Laser System Description}

The burst mode pump laser, illustrated in Fig. 1, has been described in detail previously [20,21], and will therefore be only summarized here. Briefly, a continuous wave diode pumped $\mathrm{Nd}$ :YAG ring laser serves as the primary oscillator, the output of which is pre-amplified in a double-pass variable pulse width (from $0.3-2.0 \mathrm{~ms}$ ) flashlamp-pumped pulsed amplifier. The amplifier rod is $10 \mathrm{~cm}$ in length by $6.4 \mathrm{~mm}$ in diameter, and is wedged at a few degrees to mitigate self lasing. The resulting smooth pulse is formed into a "burst" train using a custom, dual Pockels cell "slicer," the output of which is further amplified in a series of four additional flashlamp pumped amplifiers, with $6.4 \mathrm{~mm}, 6.4 \mathrm{~mm}, 9 \mathrm{~mm}$, and $12.7 \mathrm{~mm}$ diameter $\times 10 \mathrm{~cm}$ long rods, respectively. This configuration is somewhat different from the seven amplifier configuration which has been used previously for operation at burst frequencies of up to $1 \mathrm{MHz}$ [22]. As will be discussed below, the desire to obtain the highest possible spatial resolution dictated the use of a $10 \mathrm{kHz}$ frame rate intensified CMOS camera, rather than the $1 \mathrm{MHz}$ framing CCD camera used in most previous high speed studies. The lower frame rate in turn dictated a correspondingly low $(10 \mathrm{kHz})$ burst rate, and corresponding burst envelope of $1 \mathrm{~ms}$ (10 pulses at 100 microsecond spacing). Due to very significant gain refilling between the individual pulses in the burst, only five amplifiers are required to reach the high energies required for these measurements. More specifically, the slicer produces burst sequences of 8-10 pulses at 1064 $\mathrm{nm}$, each with duration of $\sim 10 \mathrm{~ns}$, and pulse energy of $\sim 10^{-9}$ Joules ( 1 nanoJoule). The remaining amplifier stages increase the individual average pulse energy to $\sim 400 \mathrm{~mJ}$ per pulse $\left(4 \times 10^{8}\right.$ gain), at $1064 \mathrm{~nm}$.

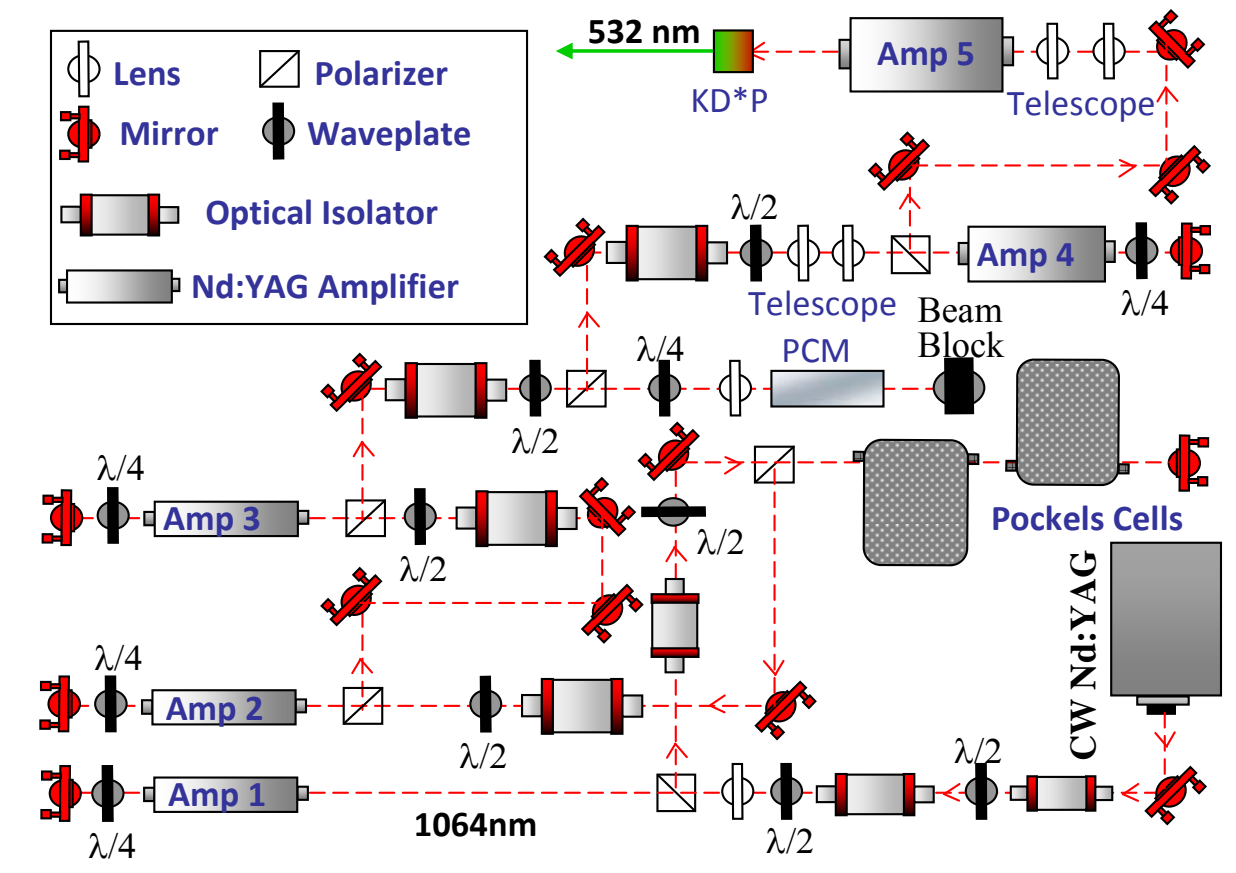

Figure 1: Schematic diagram of 5 amplifier configuration pulse burst Nd:YAG laser. 
Note that amplifiers cannot simply be added arbitrarily, as system performance is ultimately limited by the growth of Amplified Spontaneous Emission (ASE) in the forward direction. Inclusion of a Stimulated Brillouin Scattering (SBS) Phase Conjugate Mirror (PCM) is a well known approach for improving the spectral characteristics of a pulsed laser beam [23]. For this work, the purpose of the PCM is to both improve the on/off contrast of the pulse slicer and to limit ASE in the forward direction. Fluorinert FC-75, which has been used extensively as an SBS fluid [18] and has previously performed well when operating the burst mode laser at less than $\sim 250 \mathrm{kHz}$ [18], was used for this work.

The fundamental output at 1.06 microns is converted to second $(532 \mathrm{~nm})$ and third $(355 \mathrm{~nm})$ harmonic wavelengths using a pair of KD*P crystals. The third harmonic is then used as the pump for a home-built injection-seeded optical parametric oscillator (OPO) system. The OPO output is sum-frequency mixed with residual $355 \mathrm{~nm}$ from the pump laser, creating highfrequency bursts of tunable output in the vicinity of $226 \mathrm{~nm}$, which are used for NO PLIF imaging. The repetition rate of the burst sequence can be as high as $10 \mathrm{~Hz}$, but is typically limited to $1-4 \mathrm{~Hz}$ when operating with burst envelopes exceeding $\sim 0.5 \mathrm{~ms}$, due to thermal loading of the amplifier rods. Figure 2 shows a typical 10-pulse oscilloscope trace obtained at 10 $\mathrm{KHz}$ burst rate at $532 \mathrm{~nm}$. The average individual pulse energy is $\sim 150 \mathrm{~mJ}$. Note that the trace was obtained with a relatively low sampling rate oscilloscope that required the detector output to be deliberately broadened in time (using a load resistor and the intrinsic cable capacitance).

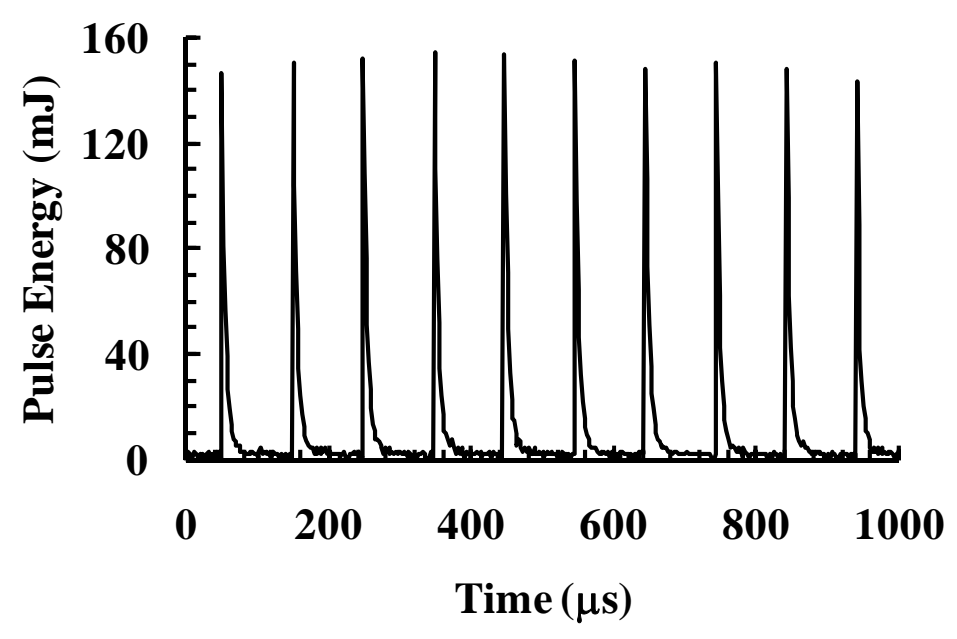

Figure 2: A typical ten-pulse laser burst trace at $532 \mathrm{~nm}$ with 100 microsecond inter-pulse spacing ( $10 \mathrm{kHz}$ repetition rate). Average pulse energy is $\sim 150 \mathrm{~mJ}$.

For spectrally narrowed imaging the OPO was operated with injection seeding [18] using an external cavity diode laser (ECDL) manufactured by Sacher, Inc. The OPO "idler" beam is seeded, which results in line-narrowed output for both the idler and signal waves. For the seeded measurements performed in this work the seed laser was tuned to $822.044 \mathrm{~nm}$ (vacuum wavelength - measured with a HighFinesse WS-6 Fizeau interferometer wavemeter), which after mixing with the third harmonic beam produced UV output at $226.244 \mathrm{~nm}$, exciting the $\mathrm{Q}_{1}(5)$ transition of the NO A-X $(0,0)$ band. This transition was selected based on the fact that the nominal static temperature of the fuel plume was predicted to vary between $\sim 100 \mathrm{~K}$ at the nozzle 
exit (ie, the near wall region of the jet) to $\sim 400 \mathrm{~K}$ after mixing with the free stream flow. Figure 3 shows a set of $226 \mathrm{~nm} 10 \mathrm{KHz}$ burst traces. The average individual pulse energy is $\sim 0.2 \mathrm{~mJ} /$ pulse.

The UV beam had a circular cross section with a $5 \mathrm{~mm}$ diameter, and it was directed upwards, using a series of mirrors, to the top of the facility. After propagation $(\sim 5$ meters $)$ to the shock tunnel, however, the beam had diverged to a nominally $10 \mathrm{~mm}$ diameter. The laser beam was then formed into a thin sheet using the combination of a cylindrical lens and a spherical lens. A positive $300 \mathrm{~mm}$ focal length cylindrical lens focused one axis of the beam which then propagated $600 \mathrm{~mm}$ and passed through a 1 meter focal length, $50 \mathrm{~mm}$ diameter spherical lens. This pair of lenses increased the total width of the beam to $\sim 100 \mathrm{~mm}$. Of this $100 \mathrm{~mm}$ the central $50 \mathrm{~mm}$ was used for the actual imaging. Using only the central two inches resulted in improved sheet uniformity and also provided some accommodation to tunnel motion, which was known to translate parallel to the primary flow axis during facility operation. Since the beam delivery optics were not fixed with respect to the tunnel, this motion could, in principal, result in clipping of the sheet at the facility entrance window. In practice this was not found to be a significant issue. The final UV beam was $\sim 0.5 \mathrm{~mm}$ thick.

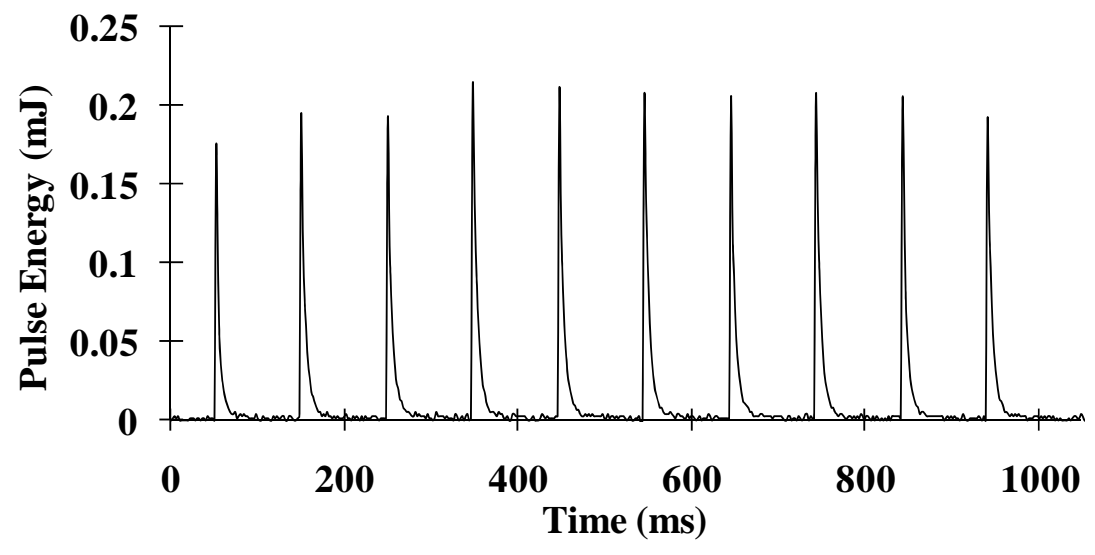

Figure 3: A typical ten-pulse laser burst trace at $226 \mathrm{~nm}$ with 100 microsecond inter-pulse spacing. Average pulse energy is $\sim 0.2 \mathrm{~mJ}$.

\subsection{8” Shock Tunnel Facility and Pulse Burst Laser Facility Integration}

The CUBRC 48 inch shock tunnel facility is the smallest of three such hypersonic facilities located at CUBRC in Buffalo, N.Y., which can provide duplicated velocity/altitude conditions for combustion studies. Fundamental hypervelocity studies from high-enthalpy re-entry physics to interceptor flow dynamics and turbulent combustion chemistry in full-scale combustion ducts have been studied with these facilities. The 48 inch Shock Tunnel can generate total enthalpy conditions in excess of $4 \mathrm{MJ} / \mathrm{kg}$ required for true temperature combustion studies at Mach 8 . The steady state test times of 5 to 10 milliseconds at this high enthalpy condition provide sufficient flow lengths to stabilize complex interaction regions and the combusting flows behind angled wall jets in a flight scale (HIFiRE II) combustor. With different contoured-nozzles and throats, the 48-inch Shock Tunnel can support test flow in the Mach number range from 6 to 20. For the proof of concept imaging experiments described in this work, a nominal Mach 9 nozzle/throat configuration was used to produce the inlet test gas. Figure 4 shows a photograph of the shock tunnel, with the high frame rate imaging system installed. 


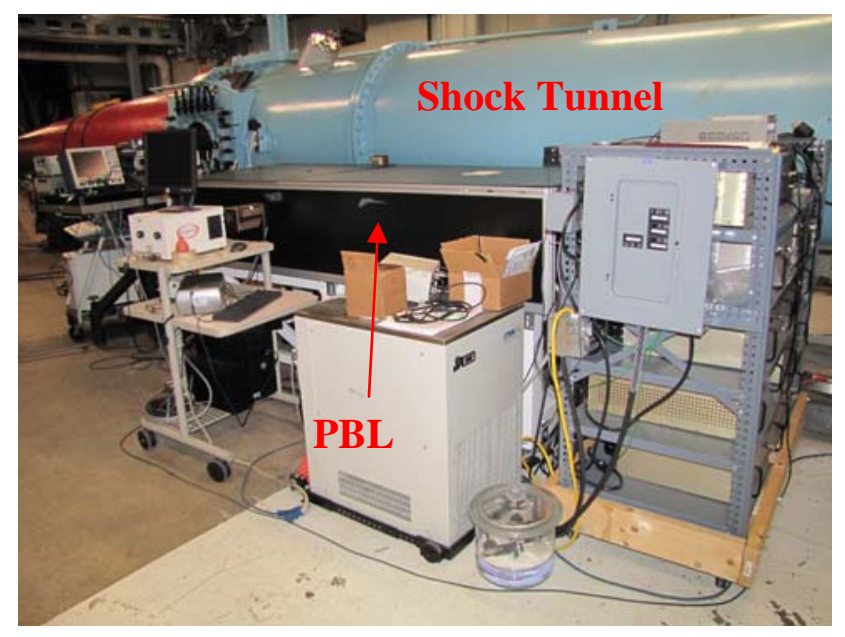

Figure 4: Photograph of CUBRC 48" Shock Tunnel with Pulse Burst Laser Installed.

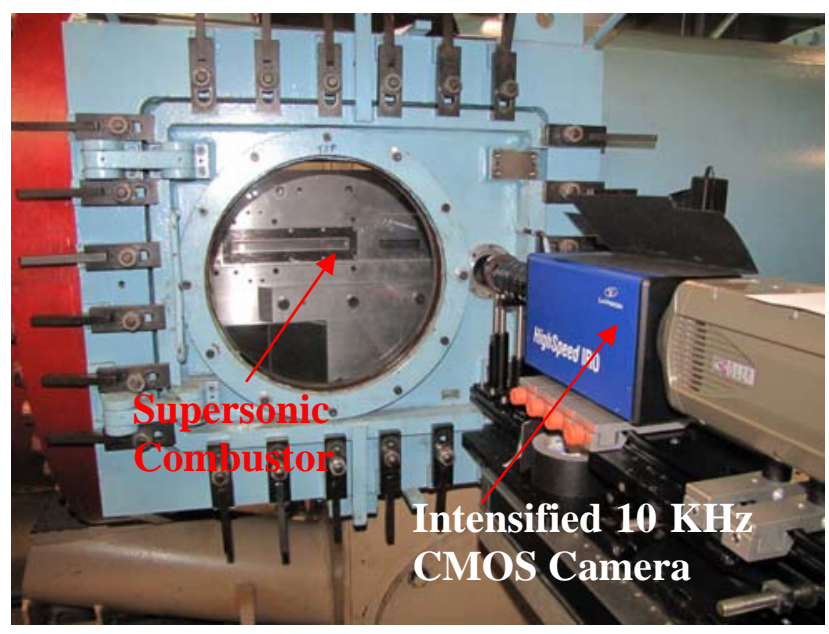

Figure 5: Photograph of generic Scramjet engine model and Intensified CMOS camera system.

The MURI combustion duct is shown supported in the test section of the 48-inch Shock Tunnel in Figure 5. This combustion duct has dimensions of $40 \mathrm{~cm}$ (height) $\times 40 \mathrm{~cm}(\mathrm{span}) \times$ $200 \mathrm{~cm}$ (length). The rectangular combustion section within this model is approximately $10 \mathrm{~cm}$ (span) x $3 \mathrm{~cm}$ (height) $\times 30 \mathrm{~cm}$ in length where the Mach number is approximately 3 based on CFD computations. Five $0.254 \mathrm{~cm}$ diameter sonic fuel injectors are located on the bottom wall, spaced $6 \mathrm{~mm}$ apart and oriented at 30 degrees with respect to the model floor. All five injectors are fuelled. These injectors operate at an injection pressure of nominally 500 psi. For all images to be shown below, the injected gas is $5 \%$ nitric oxide (NO) in helium, which is selected as a non-combustible surrogate for hydrogen, in order to study mixing.

The laser optical system is mounted on a portable optical table as shown in Figure 4 so that it could be employed in a number of different wind tunnel facilities. A separate portable cart was assembled for the flashlamp power supplies and other electrical driver components. The power supplies were individually fused to two standard three-phase $220-\mathrm{V}, 30$-amp breaker boxes. The UV output (shown in Fig. 6) was directed through two fused silica windows, located at the top of the facility and test section, respectively. The downward propagating laser sheet was focused directly on the bottom surface of the combustor (there was no exit window). A $10 \mathrm{KHz}$ intensifier (LaVision HS-IRO) and CMOS camera (Vision Research, Phantom 710) (as shown in Fig. 5) was placed close ( $\sim 6$ inches) to the test section imaging window, resulting in the object plane being approximately 0.75 meters from the camera lens. The total camera pixel format was $800 \times 1200$. As alluded to earlier, this camera was utilized in order to maximize obtainable spatial resolution while simultaneously maintaining an acceptable field of view. This camera was used in combination with a singlet plano-convex UV lens (200 mm nominal focal length, 50 $\mathrm{mm}$ diameter) for imaging, which was selected due to the lack of an available long focal length multi-element UV lens. The resulting nominal object plane spatial scale was $\sim 75$ microns/pixel, with actual spatial resolution limited in practice by the LaVision intensifier and the performance of the plano-convex lens. This imaging was accomplished using a nominal $3.4 \times$ magnification, resulting in a total object plane field of view of $\sim 60 \mathrm{~mm}$ (vertical) $\times 85 \mathrm{~mm}$ (horizontal) for the full $800 \times 1200$ pixel format sensor. Note that the intensifier diameter is $25 \mathrm{~mm}$ and the solid 
collection angle is $\sim \mathrm{f} / 15$, which was sufficient for high signal/noise instantaneous NO PLIF imaging.

Use of the available higher frame rate ICCD camera (Princeton Scientific Instruments, PSIIV), which has a pixel format of $160 \times 160$, would have resulted in a spatial resolution degraded by a factor of approximately ten. Since it was initially estimated that the penetration of the injector plume into the free stream flow would in some cases be only on the order of 1-3 mm, it was decided to opt for maximum spatial resolution at the expense of frame rate.

During the shock tunnel experiments, the intensifier gain was fixed at $\sim 50 \%$ of its maximum, and the exposure gate set to $\sim 500 \mathrm{~ns}$. A custom made 2-inch diameter transmission filter from Layertec, $\mathrm{GmbH}$ was placed in front of the plano-convex lens to efficiently block the laser's scattering wavelength while transmitting most of the red-shifted fluorescence (transmit $<1 \% @ 226 n m$ and >80\%@235-280nm).

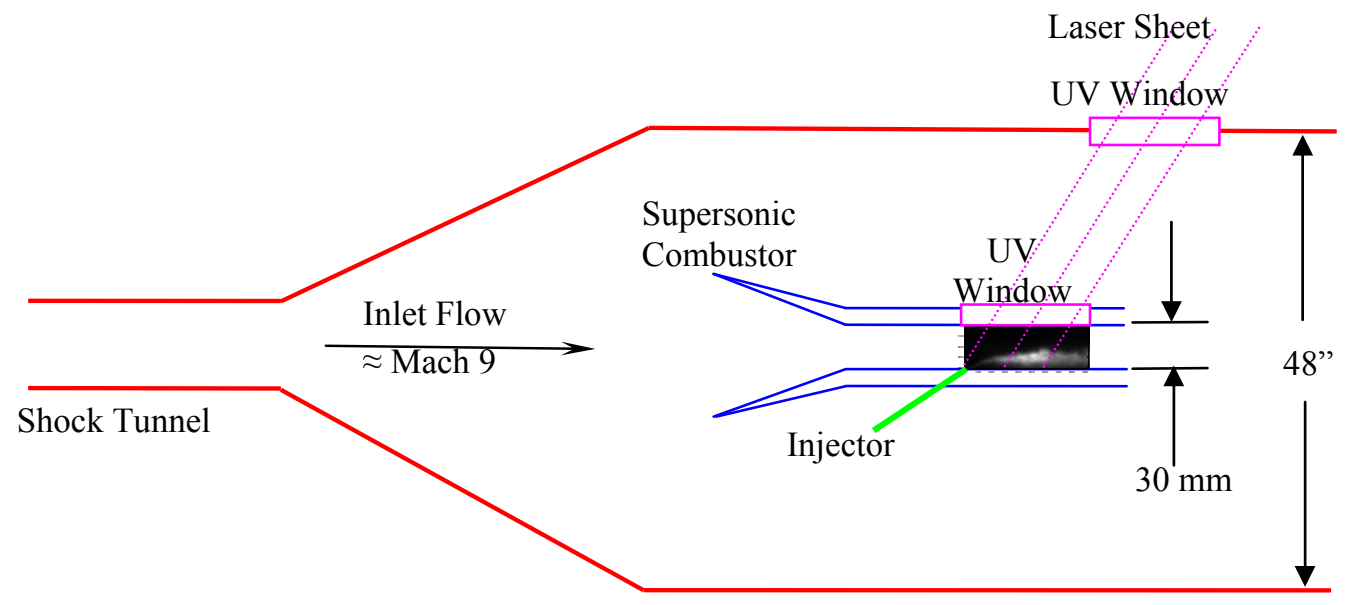

Figure 6: Simplified schematic diagram illustrating the combustion duct and laser propagation geometry. The inlet flow is nominally Mach 9 and the injection angle is approximately 30 degrees.

Due to the steady state test time of $\sim 5-10 \mathrm{~ms}$, a somewhat complicated scheme was required in order to synchronize the tunnel run with the laser and camera systems. In particular the CMOS camera system required three distinct synchronization signals, and the pulse burst laser needed to be continuously triggered at $1 \mathrm{~Hz}$ during warm-up prior to the tunnel run, mostly due to the OPO and sum-frequency mixing crystals which were not temperature stabilized. The timing box labeled "SRS Delay Generator 1" (near the center of Fig. 7) was used to generate this $1 \mathrm{~Hz}$ system trigger. It triggered a second SRS Delay generator, which in combination with two other delay generators provided triggers for the laser flashlamp power supplies and the pulse forming pulse slicer. This aspect of the synchronization/triggering was unchanged from our previous work. The Phantom CMOS camera required a continuous $10 \mathrm{kHz}$ TTL "clock" pulse, as well as a separate $10 \mathrm{KHz}$ burst for triggering the actual image acquisition series. The LaVision intensifier required a second $10 \mathrm{kHz}$ burst for synchronization to the CMOS. The $10 \mathrm{kHz}$ burst output of the primary SRS delay generator was inhibited with a 99.9 percent duty cycle (1 ms out of each 1 second cycle) during system warm-up (labeled "Warm Up Inhibit.") Just prior to a 
tunnel run this inhibit timing was switched to a second timing box (labeled "Shock Tunnel) which was triggered from a pressure transducer signal indicating that the primary flow had been established. In this manner the $1 \mathrm{~Hz}$ warm-up was maintained for all but one pulse prior to the facility firing, and even this was manually manipulated to give a near $1 \mathrm{~Hz}$ interval between the warm-up trigger signals and that of the facility. Note that this procedure resulted in a maximum of $+/-50$ microsecond jitter between the triggering of the camera system and the start of acquisition, which is negligibly small compared to the facility run time of $5-10 \mathrm{~ms}$.

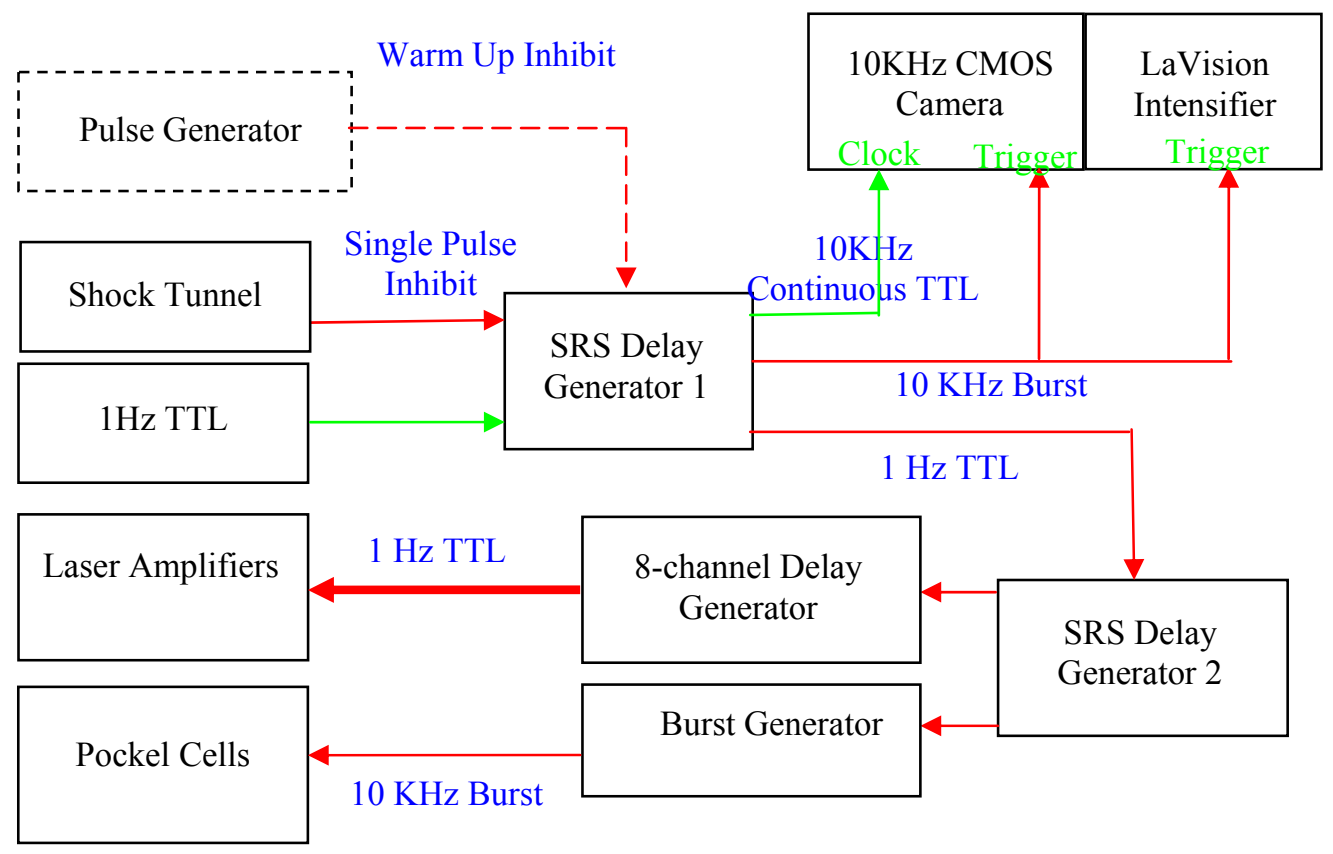

Figure 7: Block diagram illustrating triggering employed for the shock tunnel measurements.

\section{ILLUSTRATIVE IMAGING RESULTS}

\subsection{Pre-Facility Run Under-Expanded Jet Images}

In this section we present a sampling of illustrative high frequency frame rate imaging results at $10 \mathrm{kHz}$. Prior to obtaining images from shock tunnel runs, sets of images were obtained from the model in the identical geometry to that which would be used for tunnel runs but with the injector operating as a highly under-expanded jet, (i.e., emptying into a nominal vacuum without a main flow). This provided a convenient means to align the optical system, determine adequate NO seed and intensifier gain levels, test the triggering and warm-up procedures, and also to provide an approximate assessment of the image quality. Figure 8 shows a typical six frame sequence of highly under expanded jet images obtained from a single $10 \mathrm{kHz}$ laser burst. The flow is $\mathrm{NO} /$ helium supplied through the 5 sonic orifices with a 500 psi plenum pressure. The laser sheet interrogates the flow out of a single orifice. The field of view is $3.0 \mathrm{~cm} \times 6.5 \mathrm{~cm}$, with corresponding spatial scale of $\sim 75$ microns/pixel. The flow direction is from left to right. While admittedly qualitative, the image quality is quite reasonable, illustrating the well known, 
and rather complex, structure of the underexpanded jet. In particular the characteristic "barrel" shock is clearly evident, as is some irregular - possibly turbulent - structure, particularly downstream of the Mach disk (normal shock).

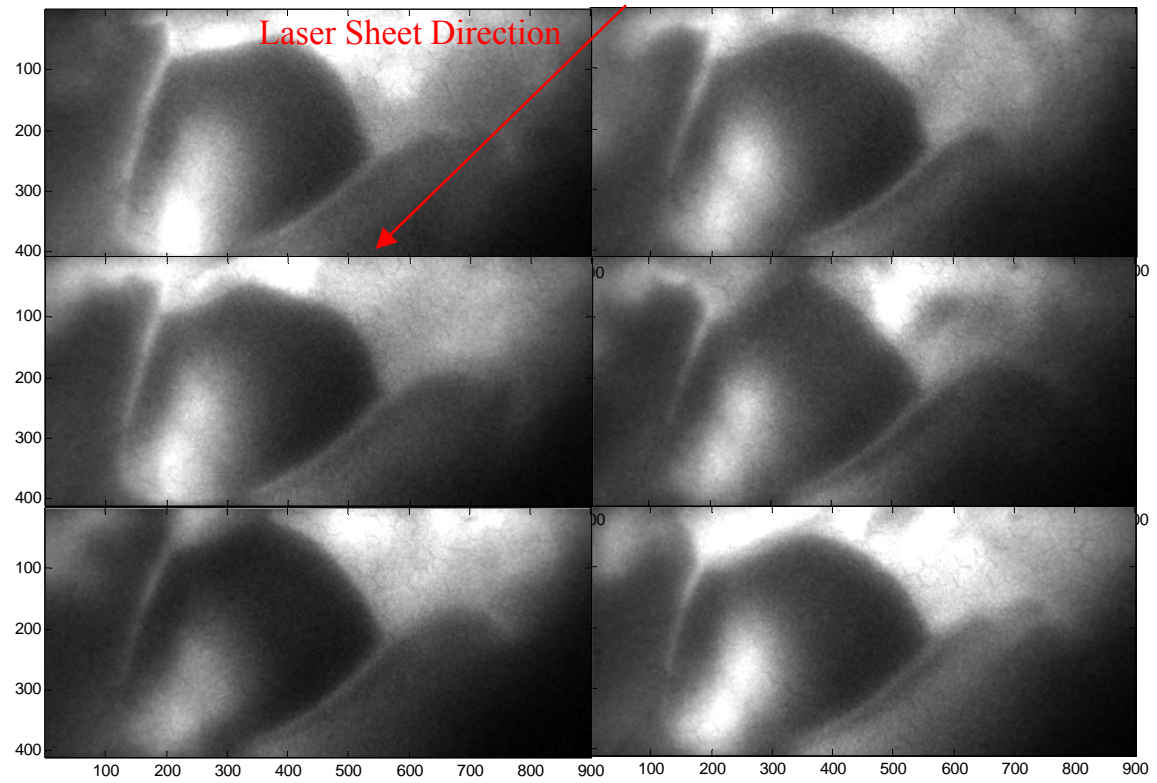

Figure 8: Sequence of 6 under-expanded jet images from 5\% NO seeded helium fuel injector, obtained from single laser burst at $10 \mathrm{kHz}$. Injector pressure is $500 \mathrm{psi}$, expanded into nominal vacuum. Imaged field of view is $3.0 \mathrm{~cm} \times 6.5 \mathrm{~cm}$. Laser sheet propagation direction is $\sim 45$ degrees, as indicated.

\subsection{Facility Run Images}

Figure 9 shows an example eight-frame image sequence (top left to bottom right) obtained at $10 \mathrm{kHz}$ frame rate from a single facility run at nominal free stream Mach number of 9 . The field of view is again $\sim 3.0 \mathrm{~cm} \times 6.5 \mathrm{~cm}$ and the nominal free stream flow velocity is $\sim 1200 \mathrm{~m} / \mathrm{s}$, with flow direction from left to right. The nominal static temperature and pressure at the exit of the fuel injector is $\sim 100 \mathrm{~K}$ and $\sim 75 \mathrm{kPa}$ (570 Torr), respectively, based on CFD simulation (See Fig. 11). The corresponding conditions of the primary flow in the duct are $\sim 600 \mathrm{~K}$ and $30 \mathrm{kPa}(230$ Torr), respectively, so that in this instance the jet is much closer to pressure matched than that shown in Fig. 8. The injected jet then expands rather rapidly, with the static pressure dropping and the temperature rising (due to mixing with the free stream) in the plume to $\sim 20 \mathrm{kPa}$ and 500 $\mathrm{K}$, respectively (again according to CFD simulation), by the right most portion of the image.

The downward propagating laser sheet was brought incident to the facility from top right to bottom left, at an angle of $\sim 45^{\circ}$, as shown in Fig. 6 . The primary flow test gas is pure nitrogen and the injector test gas, as for the case shown in Fig. 8, is 5\% NO in helium at 500 psi injection pressure. The gas is injected from the bottom left corner at $30^{\circ}$ injection angle with respect to the free stream flow direction. Note that the images shown in Fig. 8 have been cropped to display only $\sim 400$ (height) $\times 900$ (horizontal) pixels. The average intensity in detector "counts" is $\sim 1000-3000$ out of a total quantum well of 4,096, which was achieved using an intensifier gain of approximately $\sim 50 \%$ of its maximum. The corresponding background noise (mostly detector read noise) is $\sim 20$ counts. 
From Fig. 9 we can see that the fuel plume injection rapidly spreads and flaps, filling approximately half of the total $30 \mathrm{~mm}$ cavity height after only a few centimeters of downstream propagation. In actual operation both floor and top injector banks would be utilized, which would result in a combustion chamber which is reasonably mixed at this point. It can be seen, however, that the mixing process is highly turbulent, as would be expected. While it cannot be determined definitively from planar imaging, it appears that the plume spreads rapidly, and turbulently, in three dimensions. Of course since the flow speed is in excess of $1 \mathrm{~km} / \mathrm{sec}$, the images in Fig. 10 are temporally uncorrelated at the $10 \mathrm{kHz}$ frame rate in which they were acquired. The uniformity of the laser sheet is also a potential issue when interpreting these images, but based on the images in Fig. 8, and recalling that the laser sheet is propagating downward at $\sim 45$ degrees, it appears that sheet non-uniformity is not enormously significant. As stated previously, only the central two inches of a nominally four inch wide sheet was used for the actual imaging. In future experiments it may be possible to measure the intensity distribution of the laser sheet on a pulse-to-pulse basis.

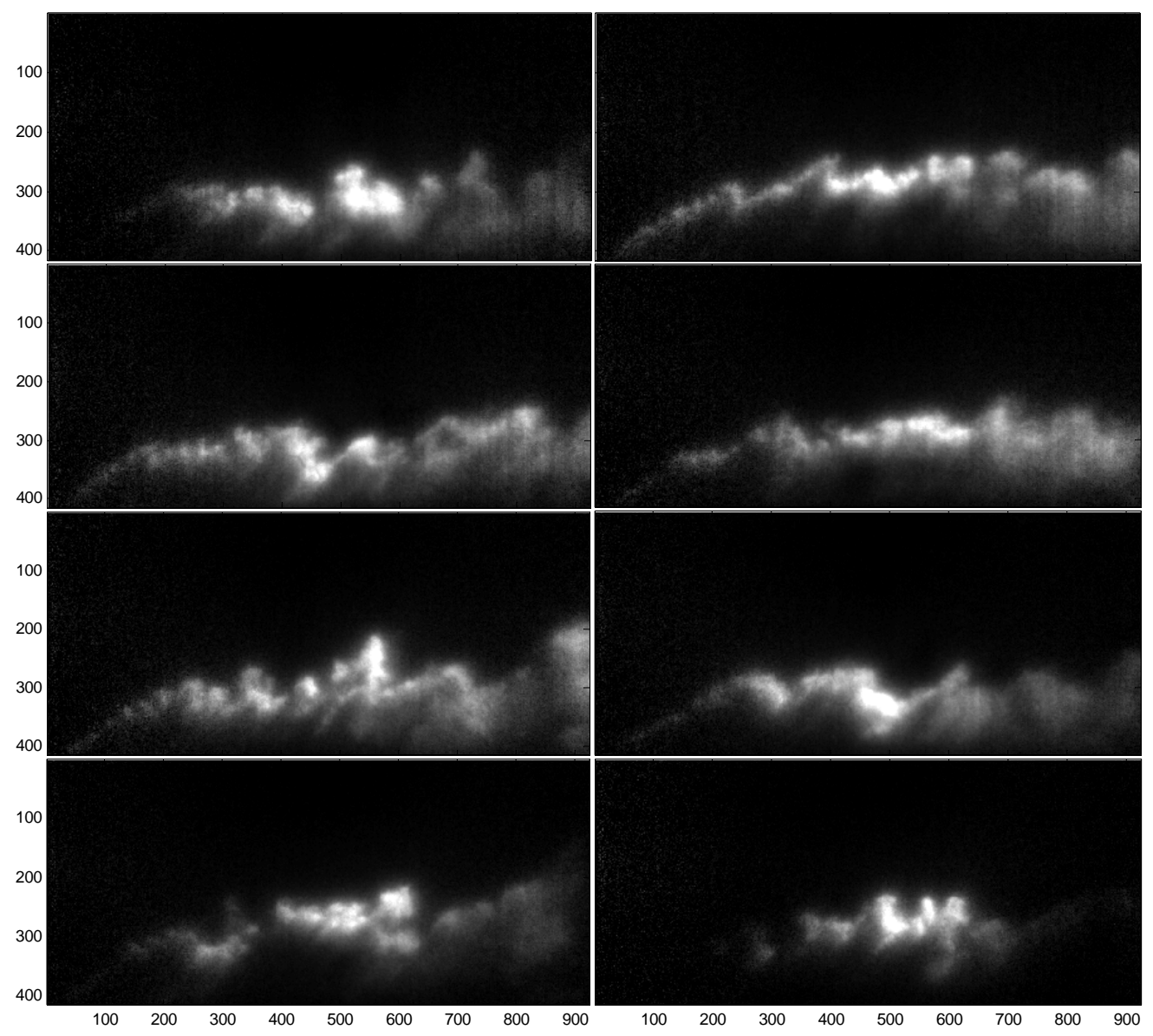

Figure 9: $10 \mathrm{KHz}$ NO PLIF images of flow in a supersonic combustor cavity. Injected gas is 5\% $\mathrm{NO}$ in Helium at 500 psi pressure. Test gas is pure $\mathrm{N}_{2}$. Injector angle is 30 degrees. The numbers on the axes indicate pixels. Field of view is $\sim 3.0 \mathrm{~cm} \times 6.5 \mathrm{~cm}$. 
An underexpanded jet structure, similar to but much smaller than that shown in Fig. 8 was expected to be observed in Fig. 9, near the bottom-left side of each image. No such structure was observed. Three different factors may contribute to prevent to this structure from being observed. First, the laser energy was probably lower on the edges of the images, since it approximates a Gaussian beam (it also varies pulse-to-pulse, explaining some of the image-toimage and pulse-burst to pulse-burst variation). Second, high NO concentrations are probably absorbing the laser sheet energy, preventing fluorescence near the lower wall. Based on an approximate scaling of measured absorption in a room temperature gas cell filled with a mixture of $5 \% \mathrm{NO}$ in $\mathrm{N}_{2}$, we estimate that the laser beam absorption in the current experiment could be as high as $25 \% / \mathrm{mm}$. Finally, the quenching of the fluorescence in the core of the fuel jet could be attenuating the fluorescence signal in the core of the jet more than in the flow in the duct. Since the NO injection pressure is very high, the NO-NO quenching rate is also very high, $\sim 2.4 \mathrm{x}$ $10^{8} \mathrm{sec}^{-1}$ at the $\sim 75 \mathrm{kPa}, 100 \mathrm{~K}$ condition at the injector exit, and $2.4 \times 10^{7} \mathrm{sec}^{-1}$ at center of the field of view of the jet, which has a static pressure and temperature (according to CFD) of $\sim 37$ $\mathrm{kPa}$ and $500 \mathrm{~K}$, respectively. This should be compared to the NO radiative rate of $5 \times 10^{6} \mathrm{sec}^{-1}$ ( $200 \mathrm{~ns}$ lifetime). Hence the fluorescence quantum yield $\left(\mathrm{QY}=\mathrm{k}_{\mathrm{rad}} /\left(\mathrm{k}_{\text {quench }}+\mathrm{k}_{\mathrm{rad}}\right)\right.$ varies by a factor of approximately ten over the imaged region of the plume and is $10 \mathrm{x}$ smaller in the plume than in the main flow.

Examining the fuel mixing in a time-averaged manner, the upper image in Fig. 10 is the average of 40 individual NO PLIF images obtained over several facility runs at the same flow condition. The nominal per pixel spatial scale is again $\sim 75$ microns/pixel. From this image it can be seen that fuel penetrates the primary flow at the injection angle of $\sim 30$ degrees, propagates at 30 degrees until it reaches approximately $7 \mathrm{~mm}$ in height above the floor, and then turns and propagates approximately parallel to the primary flow axis. At this point the plume also spreads rapidly, due to turbulent diffusion. After approximately 2.5 inches $(6.4 \mathrm{~cm})$ of downstream propagation the plume from the single floor injector has penetrated to just below the flow centerline.

The bottom image in Fig. 10 is a preliminary CFD simulation obtained for the same flow condition as the NO PLIF average image. The CFD was run using an unstructured RANS solver. This particular case was run steady, 3D, using the k-epsilon turbulence model with compressibility corrections, which have matched experimental data well in many cases [24]. It was run with a hybrid unstructured grid containing 3.3 million cells, and utilized half-plane symmetry in the spanwise direction of the model (ie the width of the model). Note that the actual combustor cavity expands slightly in the downstream direction, as shown in the simulation. While it is difficult to make an absolute comparison of the NO PLIF image, which has not been corrected for quenching, absorption, and laser sheet non-uniformity, and the CFD simulation, which shows NO mass fraction, it is clear that there is reasonable overall qualitative agreement. One significant discrepancy, noted above, is that the PLIF images do not show any indication of the barrel shock structure predicted by the CFD in the lower left region of the jet. As described above, the NO density is extremely high in this region and it is possible that quenching and absorption effects cause corresponding reductions in signal that mask the under-expanded jet structure. In future experiments, lower NO concentrations and weaker transitions could be used to reduce these complicating effects, thereby producing images that are easier to interpret. It appears from this preliminary comparison that the simulation shows somewhat less spreading with distance downstream than the experimental data. This could be due to inaccurate prediction of turbulent mixing and/or fuel-jet flapping. 


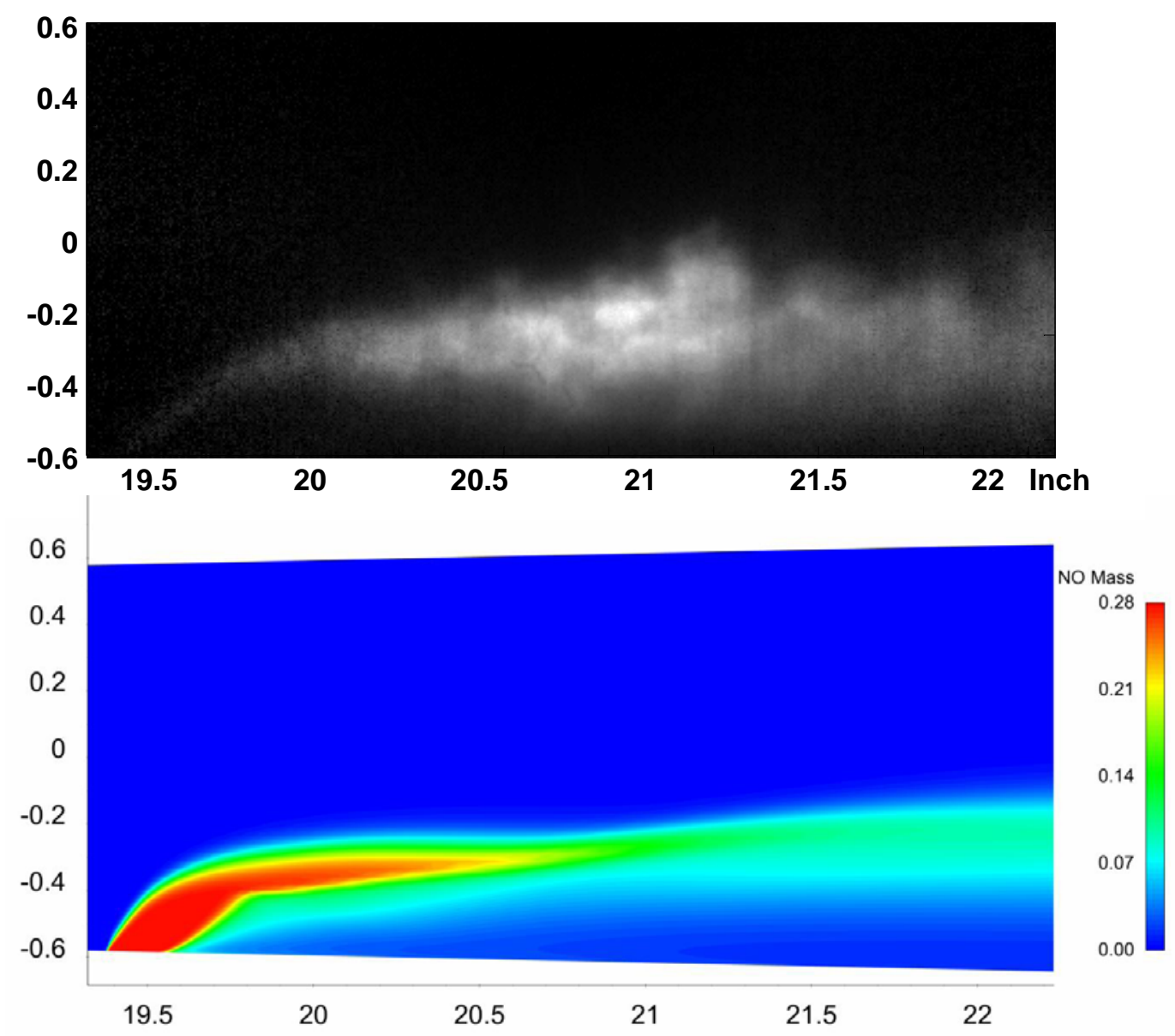

Figure 10: Comparison of an averaged NO PLIF image with CFD simulation. The axes are in units of inches.

\section{CONCLUSIONS}

Nitric oxide PLIF imaging has been demonstrated at $10 \mathrm{kHz}$ repetition rate in the CUBRC 48 inch Mach 9 hypervelocity shock tunnel using a pulse burst laser system, a custom Optical Parametric Oscillator, and an intensified CMOS camera. During the run 5\% NO in helium is injected into the combustor channel, providing a PLIF fuel tracer. Up to ten images of the jet mixing in the supersonic flow through the CUBRC MURI combustion duct were obtained during the $10 \mathrm{~ms}$ test time. High signal-to-noise images were obtained under typical free stream static temperature and pressure conditions at the combustor inlet of $400 \mathrm{~K}$ and $75 \mathrm{kPa}$ (570 Torr), respectively. Comparison with a preliminary CFD simulation shows, with some exceptions, good overall qualitative agreement between predicted NO mass fraction and the observed PLIF image intensity, averaged over forty individual images obtained during several facility runs. 
This work demonstrates the feasibility of increasing the data rate of PLIF visualization methods in high-enthalpy impulse facilities by an order of magnitude utilizing burst-mode laser technology.

\section{Acknowledgements}

The authors would like to acknowledge Ron Ungewitter at Combustion Research and Flow Technology (CRAFT Tech) for obtaining the CFD solution shown in Fig. 11. This work was supported by the Air Force Office of Scientific Research (John Schmisseur - program monitor), and a NASA Phase I Small Business Innovative Research grant to Spectral Energies, Inc. The Air Force Research Laboratory (James Gord) is also acknowledged for its ongoing contributions to the development of the laser technology essential to this work.

\section{References}

1. P.M. Danehy, S. O'Byrne, A.F.P. Houwing, J.S. Fox, and D.R. Smith, "Flow-Tagging Velocimetry for Hypersonic Flows Using Fluorescence of Nitric Oxide," AIAA Journal 41, p. 263 (2003).

2. P.M. Danehy, P. Mere, M.J. Gason, S. O'Byrne, P.C. Palma, and A.F.P. Houwing, "Fluorescence Velocimetry of the Hypersonic, Separated Flow over a Cone," AIAA Journal 39, p. 1320 (2001).

3. P.M. Danehy and S.O'Byrne, "Measurement of NO Density in a Free-Piston Shock Tunnel using PLIF," AIAA-99-0772, $37^{\text {th }}$ AIAA Aerospace Sciences Meeting, January 11-14,1999, Reno, NV.

4. S.O'Byrne, P.M. Danehy, and A.F.P. Houwing, "Nonintrusive Temperature and Velocity Measurements in a Hypersonic Nozzle Flow," AIAA-2002-2917, 22 ${ }^{\text {nd }}$ AIAA Aerodynamic Measurement and Ground Testing Conference, June 24-26, 2002, St. Louis, MO.

5. R. Hruschka, S.O'Byrne, and Harald Kleine," Two-component Doppler-shift fluorescence velocimetry applied to a generic planetary entry probe model," Exp Fluids 48, p. 1109 (2010).

6. C.-Y. Tsai, J.F. Callega, R.J. Bakos, and R.C. Rogers, "A Technique for Mixing Measuremnt in Hypervelocity Pulse Facilities using Particle Scattering Imagery." AIAA-96-2222, $19^{\text {th }}$ AIAA Aerodynamic Measurement and Ground Testing Conference, June 17-20, 1996, New Orleans, LA.

7. W.L. Roberts, M.G. Allen, R.P. Howard, G.J. Wilson, and R. Trucco, "Measurement and Prediction of Nitric Oxide Concentration in the HYPULSE Expansion Tube Facility," AIAA-942644, $18^{\text {th }}$ AIAA Aerospace Ground Testing Conference, June 20-23, 1994, Colorado Springs, CO.

8. S.D Wehe, D.S. Baer, R.K. Hanson, and K.M. Chadwich, "Measurements of Gas Temperature and Velocity in Hypervelocity Flows Using a Diode-Laser Absorption Sensor," AIAA-98-2699, $20^{\text {th }}$ AIAA Aerodynamic Measurement and Ground Testing Conference, June 15-18, 1988, Albuquerque, NM.

9. R.A. Parker, T. Wakeman, M. MacLean, and M. Holden, "Nitric Oxide Concentration Measurements with a Quantum Cascade Laser in the LENS I Hypersonic Shock Tunnel Facility," AIAA-2006-920, 44 ${ }^{\text {th }}$ AIAA Aerospace Sciences Meeting, January 9-12, 2006, Reno, NV.

10. R. Parker, T. Wakeman, M. MacLean and M. Holden. "Measuring Nitric Oxide Freestream Velocity Using a Quantum Cascade Laser at CUBRC", AIAA-2007-1329, 45 ${ }^{\text {th }}$ Aerospace Sciences Meeting \& Exhibit, Reno, NV. January 2007. 
11. M. Holden, J. Craig, A. Ratliff, and G. Sutton, "Calibration and Validation Studies in the LENS Facility," AIAA-93-2682, 2 ${ }^{\text {nd }}$ AIAA SDIO Interceptor Technology Conference, June 6-9, 1993, Albuquerque, NM.

12. M.S. Holden, R.A. Parker, "LENS Hypervelocity Tunnels and Application to Vehicle Testing at Duplicated Flight Conditions", Chapter 4, Advanced Hypersonic Test Facilities, Editors Frank Lu and Dan Marren, Volume 198, 2002.

13. P. F. Barker, A.M. Thomas, T. J. McIntyre, and H. Rubinsztein-Dunlop, "Velocimetry and Thermometry of Supersonic Flow Around a Cylindrical Body," AIAA Journal 36, 1055 (1998).

14. M.G. Allen, J.F. Cronin, S.J. Davis, R.R. Foutter, T.E. Parker, W.G. Reinecke, and D.M. Sonnenfroh, "PLIF Imaging Measurements Compared to Model Calculations in High-Temperature Mach 3 Airflow Over A Sphere," AIAA-93-0092, 31 ${ }^{\text {st }}$ AIAA Aerospace Sciences Meeting, January 11-14, 1993, Reno, NV.

15. B.K. McMillan, J.L. Palmer, and R.K. Hanson, "Temporally resolved, two-line fluorescence imaging of NO temperature in a transverse jet in a supersonic cross flow," Appl. Opt 32, p. 7532 (1993).

16. M.J. Dyer and D.R. Crosley, "Two-dimensional imaging of $\mathrm{OH}$ laser-induced fluorescence in a flame," Opt. Lett., 7, 382-384 (1982).

17. G. Kychakoff, K. Knapp, R.D. Howe, and R.K. Hanson, "Flow Visualization in Combustion Gases Using Nitric Oxide Fluorescence," AIAA J. 22, 153-154 (1984).

18. N. Jiang, W. Lempert, G. Switzer, T. Meyer and J. Gord, "A narrow-linewidth MHz repetition-rate optical parametric oscillator for high-speed flow and combustion diagnostics," Appl. Opt. 47, 6471 (2008).

19. N. Jiang and W. Lempert, "Ultra-high frame rate Nitric Oxide Planar Laser Induced Fluorescence imaging," Optics Letters, 33, (19), 2236-2238, 2008.

20. N Jiang, M. Webster and W. Lempert, "New advances in generation of high repetition rate burst mode laser output," Applied Optics, 48, (4), B23-B31, 2009.

21. B. Thurow, N. Jiang, M. Samimy, and W.R. Lempert, "A Narrow Linewidth MHz Rate Pulse Burst Laser for High-Speed Flow Diagnostics,” Applied Optics, 43, (26), 5064-5073, 2004.

22. N. Jiang, M. Webster, W. R. Lempert, J. D. Miller, T. R. Meyer, C. B. Ivey, and P. M. Danehy, "MHz-Rate NO PLIF Imaging in a Mach 10 Hypersonic Wind Tunnel," Applied Optics 50, No. 4, 1 February 2011.

23. C. K. Ni and A. H. Kung, "Effective suppression of amplified spontaneous emission by stimulated Brillouin scattering phase conjugation," Optics Letters, 21, 1673-1675 (1996).

24. R.J. Ungewitter, K.W. Brinckman, and S.M. Dash, "Advanced Modeling of New Fuel/Air Mixing Data Sets for Scramjet Applications," 45 ${ }^{\text {th }}$ AIAA/ASME/SAE/ASEE Joint Propulsion Conference \& Exhibit, Denver, CO, August 2-5, 2009. 